\title{
Efeito do Estímulo Mamário Sobre o Índice de Bishop em Gestações a Termo
}

\author{
Effect of Nipple Stimulation on Bishop Scores in Term Pregnancies
}

Alexandre Faisal Cury, Carlos Augusto Vieira de Moraes

\section{RESUMO}

Objetivo: avaliar se o estimulo mamário realizado por primigestas, a partir de 40 semanas de idade gestacional, modifica o indice de Bishop.

Método: foram estudadas 64 primigestas, sem intercorrências clinicas e/ou obstétricas, divididas em dois grupos. Um deles, chamado de grupo do estímulo mamário (E.M.), era composto por 29 gestantes e o outro, chamado de grupo controle (G.C.), por 35 gestantes. $O$ E.M. foi orientado a realizar a estimulação do mamilo bilateralmente, em "corda de relógio", com duração de dois minutos de estímulo e cinco de repouso, durante 30 minutos. O estímulo foi realizado três vezes ao dia até completarem-se as 41 semanas de idade gestacional ou a gestante entrar em trabalho de parto. Para análise estatística das diferenças encontradas, utilizou-se o teste $t$ de Student, com nivel de significância de 5\%.

Resultados: uma vez realizado o estímulo mamário no grupo experimental, comparações foram realizadas com o grupo controle, levando-se em conta a época do parto. Os resultados mostraram não haver diferença significativa entre os grupos, no que concerne ao amadurecimento do colo de acordo com o indice de Bishop.

Conclusões: não houve diferença no indice de Bishop em primigestas, com 40 semanas de idade gestacional, submetidas ao estímulo mamário, quando comparadas ao grupo controle.

PALAVRAS-CHAVE: Teste do estímulo mamário. Indução do parto. Gravidez normal.

\section{Introdução}

Em 1964, Bishop propôs um sistema para predizer o sucesso da indução do parto baseado no estado do colo uterino prévio à indução. Assim,

Maternidade Dr Cury - Osasco - São Paulo

Correspondência:

Alexandre Faisal Cury

Rua Dr Mário Ferraz 135/42 - J. Europa

01453-010 - São Paulo - SP mulheres com cérvix "favorável" teriam menor período de latência, menor duração do trabalho de parto e maior possibilidade de sucesso em sua indução em relação à outras, avaliadas como portadoras de colo "desfavorável"2.

Ao longo dos anos, vários métodos têm sido propostos para o "amadurecimento" da cérvix e dentre eles o estímulo mamário (E.M.). A estimulação do mamilo e da aréola desencadeia impulsos aferentes nos núcleos supra-ópticos e paraventriculares do hipotálamo. Impulsos 
eferentes promovem a liberação de ocitocina pela hipófise posterior. A ocitocina atua nos órgãos-alvo: na mama, promove a apojadura do leite, no útero, desencadeia contrações.

A maturação do colo tem indicação em grande número de intercorrências obstétricas. No entanto, dispomos em nosso meio de reduzido arsenal terapêutico para este fim. A utilização do estímulo mamário, método natural, prático e sem ônus econômico, se revela alternativa muito interessante.

Nosso objetivo foi estudar as mudanças no Índice de Bishop em gestantes de 40 semanas submetidas ao estímulo mamário.

\section{Pacientes e Métodos}

No período de janeiro de 1995 à junho de 1996, foram avaliadas 64 primigestas, clínica e obstetricamente normais, em acompanhamento pré-natal em clinica privada dos autores, divididas em dois grupos. O primeiro, com 29 gestantes, denominado grupo do estímulo mamário (E.M.) e o segundo, com 35 gestantes, denominado grupocontrole (G.C.). Todas as pacientes foram orientadas quanto aos propósitos da pesquisa, com que consentiram verbalmente.

Ambos os grupos eram constituídos por primigrávidas com 40 semanas de gestação. A idade gestacional foi calculada pela data da última menstruação e confirmada por ultra-sonografia obstétrica precoce (antes de 20 semanas de gravidez). Quando atingiam as 40 semanas, todas as clientes foram submetidas à U.S.G. obstétrica para avaliação da quantidade de líquido amniótico e monitoragem fetal (cardiotocografia basal). Comprovada a boa vitalidade fetal seguiam o presente protocolo, repetindo a monitoragem em dias alternados. Nesta ocasião (40 semanas) todas as gestantes eram submetidas ao exame obstétrico de rotina e toque para avaliação do índice de Bishop, pelo mesmo obstetra.

O grupo do E.M. foi então orientado a realizar a estimulação mamária bilateral, em "corda de relógio", com duração de dois minutos de estimulo e cinco minutos de repouso, durante trinta minutos. O estímulo era realizado três vezes ao dia (manhã, tarde e noite) até completarem-se as 41 semanas ou a gestante entrar em trabalho de parto. Já o grupo controle foi orientado a não realizar qualquer manipulação mamária neste período.

Para avaliação do índice de Bishop (I.B.), seguimos protocolo definido, segundo o qual índice de 0-3 foi considerado como "não-maduro", 4-7 "moderadamente maduro" e acima de 7 "maduro"4. O índice de Bishop foi reavaliado com 41 semanas completas ou quando a gestante entrava em trabalho de parto. Nestas duas ocasiões, estudouse a distribuição das mulheres conforme a melhora no indice de Bishop, nos dois grupos.

Para a análise estatística das diferenças encontradas, utilizou-se o teste t de Student, com nivel de significância de $5 \%$.

\section{Resultados}

O estudo estatístico das variáveis sóciodemográficas nos dois grupos mostrou que a maioria das gestantes pertenciam à faixa etária de 20 a 24 anos (58, 7\% E.M. e 52, 2\% G.C.). Comparando-se os grupos, não houve diferença estatisticamente significante entre eles, quanto a participação em cada faixa etária $(\mathrm{t} \leq 1,01 ; \mathrm{p}>0,10)$ (Tabela 1).

Tabela 1 - Distribuição das grávidas do grupo do estímulo mamário (E.M.) e do grupo controle (G.C.) por faixas etárias.

\begin{tabular}{lrrrrr}
\hline \multirow{2}{*}{$\begin{array}{l}\text { Idade } \\
\text { em anos }\end{array}$} & \multicolumn{2}{c}{ E.M. } & \multicolumn{2}{c}{ G.C. } \\
\hline Menos de 15 & 1 & 3,4 & $n$ & $\%$ \\
15 à 19 & 3 & 10,3 & 0 & - \\
20 à 24 & 17 & 58,7 & 5 & 17,2 \\
25 à 29 & 7 & 24,2 & 16 & 55,2 \\
Mais de 30 & 1 & 3,4 & 6 & 20,7 \\
Total * & 29 & 100,0 & 2 & 6,9 \\
\hline
\end{tabular}

* Número de mulheres com informação da idade (em 6 pacientes do grupo controle a idade era ignorada)

NS $(t \leq 1,01 ; p>0,10)$ (teste $t$ de Student)

E.M. = estímulo mamário

G.C. = grupo controle

Quanto ao escore de Bishop por ocasião do exame com 40 semanas de gestação, observou-se que mais de dois terços das mulheres apresentava escore classificado como "não-maduro" (69\% E.M. e $77,1 \%$ G.C.) e que não houve diferença estatística entre os dois grupos no que se refere a esta avaliação (Tabela 2).

Igualmente, o estudo das médias do índice de Bishop, na 40a semana gestacional, mostrou não haver diferença significante entre os dois grupos. No grupo E.M. a média foi de 2,62, enquanto no G.C. foi de 2,63 ( $p>0,10$ ) (Tabela 3).

Após a estimulação mamária no grupo experimental foram realizados estudos 
comparativos levando-se em conta a época do parto. A Tabela 4 mostra a distribuição das mulheres segundo os indices de Bishop, nos grupos, por ocasião do parto. Observou-se que, houve maior participação das mulheres do grupo do E.M. na categoria moderadamente maduro (E.M. 41,4\%, G.C. $31,4 \%$, ao passo que o grupo controle teve maior número de mulheres na categoria maduro (G.C. $31,4 \%$, E.M. 20,7). Estas diferenças, no entanto, não foram estatisticamente significativas.

Tabela 2 - Distribuição das mulheres segundo os índices de Bishop, na 40a semana

\begin{tabular}{lrrrr}
\hline Índice de Bishop & \multicolumn{3}{c}{ E.M. } & $\begin{array}{r}\text { G.C. } \\
\end{array}$ \\
& $\mathrm{N}$ & $\%$ & $\mathrm{~N}$ & $\%$ \\
\hline$\leq 3$ & & & & \\
(Não maduro) & 20 & 69,0 & 27 & 77,1 \\
$4-7$ & & & & \\
(Moderadamente maduro) & 9 & 31,0 & 7 & 20,0 \\
$\leq 8$ & & & & \\
(Maduro) & 0 & 0 & 1 & 2,9 \\
Total & 29 & 100 & 35 & 100 \\
\hline
\end{tabular}

NS $(p>0,05)$ (teste $t$ de Student)

E.M. = estímulo mamário

G.C. $=$ grupo controle

Tabela 3 - Média \pm desvio padrão do índice de Bishop, nas grávidas do grupo do estímulo mamário (E.M.) e do grupo controle (G.C.), na 40as semana.

\begin{tabular}{lcc}
\hline & E.M. & G.C. \\
\hline Média \pm DP & $2,62 \pm 1,59$ & $2,63 \pm 1,92 *$ \\
Total de pacientes & 29 & 35 \\
\hline
\end{tabular}

${ }^{*} t=0,025, p>0,10$ (teste $t$ de Student)

E.M. = estímulo mamário

G.C. = grupo controle

Tabela 4 - Distribuição das mulheres grávidas do grupo do estímulo mamário (E.M.) e do grupo controle (G.C.), segundo o índice de Bishop, na ocasião do parto

\begin{tabular}{|c|c|c|c|c|}
\hline \multirow[t]{2}{*}{ Índice de Bishop } & \multicolumn{2}{|c|}{ E.M. } & \multicolumn{2}{|r|}{ G.C. } \\
\hline & $\mathrm{N}$ & $\%$ & $\mathrm{~N}$ & $\%$ \\
\hline \multicolumn{5}{|l|}{$\leq 3$} \\
\hline (Não-Maduro) & 11 & 37,9 & 13 & 37,1 \\
\hline \multicolumn{5}{|l|}{$4-7$} \\
\hline (Moderadamente & 12 & 41,4 & 11 & 31,4 \\
\hline \multicolumn{5}{|l|}{ Maduro) } \\
\hline \multicolumn{5}{|l|}{$\geq 7$} \\
\hline (Maduro) & 6 & 20,7 & 11 & 31,4 \\
\hline Total & 29 & 100 & 35 & 100 \\
\hline
\end{tabular}

NS $p<0,05$ (teste t de Student)

E.M. = estimulo mamário

G.C. $=$ grupo controle
A Tabela 5 mostra a distribuição das médias do índice de Bishop, nos dois grupos, por ocasião do parto. Observou-se que no total da amostra, independente do período em que ocorreu o parto, a média do índice de Bishop foi maior no G.C. (5.06) do que no grupo E.M. (4.59), sendo que esta diferença não foi estatisticamente significativa $(\mathrm{t}=0,66 ; \mathrm{p}>0.10)$.

A Tabela 6 apresenta a distribuição das gestantes conforme a melhora do índice de Bishop e a época do parto. Nos partos ocorridos antes de 41 semanas, 13 no E.M. e 21 no G.C., houve melhora do indice de Bishop em 20 gestantes do G.C. (95\%) e em 10 do grupo E.M. (76,4\%). Nos partos ocorridos na $41^{\text {a }}$ semana, 16 no E.M. e 14 no G.C., a melhora do índice de Bishop deu-se em $5(35,7 \%)$ das gestantes do G.C. e em $7(43,8 \%)$ das gestantes do grupo E.M. No total da amostra, independentemente da época do parto, observa-se que a melhora no indice foi mais freqüente no G.C. $(71,4 \%)$ do que no grupo E.M. $(58,6 \%)$.

Tabela 5 - Média \pm desvio padrão do índice de Bishop, nas grávidas do grupo do estímulo mamário (E.M.) e do grupo controle (G.C.), na ocasião do parto.

\begin{tabular}{lcc}
\hline & E.M. & G.C. \\
\hline Média \pm DP & $4,59 \pm 2,77$ & $5,06 \pm 2,91 *$ \\
Total de pacientes & 29 & 35 \\
\hline
\end{tabular}

$S\left({ }^{*} t=0,661, p>0,10\right)$ (teste $t$ de Student)

Tabela 6 - Distribuição das gestantes conforme a melhora do índice de Bishop, em relação à época do parto.

\begin{tabular}{lrccc} 
Melhora & \multicolumn{2}{c}{ E.M. } & G.C. \\
\hline & \multicolumn{3}{c}{ Parto antes de 41 semanas } \\
& N & $\%$ & N & $\%$ \\
\hline Sim & 10 & 76,9 & 20 & 95,2 \\
Não & 3 & 23,1 & 1 & 4,8 \\
Total & 13 & 100 & 1 & \\
\hline lo & & &
\end{tabular}

$100 \quad$ Parto na 41로 semanas

\begin{tabular}{lcccc} 
& N & $\%$ & N & $\%$ \\
\hline Sim & 7 & 43,8 & 5 & 35,7 \\
Não & 9 & 56,3 & 9 & \\
64,3 & & & & \\
\hline
\end{tabular}

Toda Semana

\begin{tabular}{lcccc} 
& $\mathrm{N}$ & $\%$ & $\mathrm{~N}$ & $\%$ \\
\hline Sim * & 17 & 58,6 & 25 & \\
71,4 & & & & \\
Não & 12 & 41,4 & 10 \\
\hline
\end{tabular}

NS $\left({ }^{*} t=1.47 ; p>0.10\right)$

E.M. = estímulo mamário

G.C. = grupo controle 
Estudando-se a significância estatística das diferenças de participação na classificação Sim (quando houve melhora) em cada uma das situações, entre os grupos, verifica-se que elas não são significantes $(t=1,47 ; p>0,10)$.

\section{Discussão}

A maturação do colo uterino é uma das preocupações mais relevantes da prática obstétrica moderna, tendo se preconizado diversas medidas para este fim. O estímulo mamário é citado na literatura como um destes recursos, contribuindo para seu emprego a praticabilidade, a facilidade e a isenção de qualquer custo econômico. No entanto, existem poucos trabalhos internacionais que se preocuparam em testar a efetividade do procedimento. Mais ainda, observa-se que o uso do estímulo mamário está mais freqüentemente associado à cardiotocografia de estresse, do que a obtenção de cervix mais favorável à indução do parto. Admite-se que o estímulo mamário possa realmente desencadear contrações, possibilitando melhor avaliação do estado de oxigenação daqueles fetos considerados suspeitos, na cardiotocografia de repouso. Diversos autores se utilizaram do E.M. para esta finalidade ${ }^{5,6,7,8}$. Ainda que a eficácia clínica do teste seja aceita, não se sabe, porém, o mecanismo determinante das contrações uterinas. Existe, de fato, controvérsia quanto ao papel da oxitocina durante o E.M. Outro trabalho cita, ainda, o emprego do E.M. como preditor do parto prematuro $^{3}$.

Na literatura internacional, Elliott \& Flaherty $^{4}$ analisaram a eficácia do método para maturação do colo uterino. Estes autores estudaram 81 mulheres na $39^{a}$ semana de gestação. No primeiro estudo, 22 foram alocadas no grupo do E.M., seguindo a mesma técnica utilizada em nosso trabalho, e 16 gestantes não realizaram qualquer estímulo, constituindo o grupo controle. $\mathrm{O}$ índice de Bishop inicial, avaliado pelo mesmo obstetra, não diferiu nos dois grupos. As participantes eram reavaliadas a cada três dias. O grupo do E.M. apresentou mudança na pontuação do índice de Bishop, da ordem de 2,8, ao passo o grupo controle apresentou variação de 0,33 no índice. Mais ainda, $50 \%$ das gestantes do grupo do E.M. entraram em trabalho de parto, contra $7 \%$ das do grupo controle. Ambos os resultados foram significativos estatisticamente.

No segundo estudo, os autores realizaram o mesmo estudo com 40 gestantes, obtendo resultados semelhantes. A melhora do indice de Bishop (E.M. = 2,0; G.C. $=0,37$ ) e o número de mulheres que entraram em trabalho de parto (E.M. $=40 \%$; G.C. $=5 \%$ ) foi igualmente significativa. As mulheres que não entraram em trabalho de parto foram distribuídas no grupo oposto e reavaliadas. Nesta análise, também houve significativa melhora do indice de Bishop (E.M. = 3,8; G.C. $=0,37$ ) e maior número de trabalhos de parto (E.M. $=26 \%$; G.C. $=11 \%)$.

Combinando os dois resultados, obtém-se no geral, melhora de 2,4 contra 0,35, no índice de Bishop no grupo do E.M.. Além disto, 45\% das mulheres neste grupo entraram em trabalho de parto contra $6 \%$ das do grupo controle.

Contrariamente, nossos resultados não são nada entusiasmadores quanto à utilização do E.M. para maturação do colo uterino. Nossas gestantes, todas primigestas, com a mesma média de idade e com idade gestacional confirmada por data confiável da última menstruação e por ultrasonografia precoce, não se beneficiaram da referida técnica. Utilizando-se o mesmo critério classificatório do colo, dos referidos autores, observa-se que a distribuição das gestantes de ambos os grupos, foi semelhante. $\mathrm{O}$ colo nãomaduro predominou nos dois grupos, o que acabaria por favorecer o emprego do E.M., caso viessem a ocorrer mudanças. Mais ainda, as gestantes submetidas ao estimulo apresentavam a mesma média do índice de Bishop, no início do estudo, que as gestantes do grupo controle, de modo que, possiveis variações no índice que viessem a se verificar pudessem ser atribuídas ao E.M. No entanto, por ocasião do parto, independentemente da semana de gestação, os critérios classificatórios do colo não mostraram diferenças entre os grupos. Neste momento, o colo mais encontrado foi o moderadamente maduro. Isto pode ser explicado pela melhora espontânea do colo com a evolução da gravidez.

A análise da média do índice de Bishop, na data do parto, não mostrou diferença significativa entre os grupos. A média no grupo do E.M., de 4,59, é semelhante à do G.C., de 5,06, atestando contra a utilidade do método.

Procurou-se também avaliar se havia ocorrido melhora do estado do colo, de acordo com a época do parto. Estudou-se, assim, esta eventual melhora, nas mulheres que tiveram o parto antes de 41 semanas ou na 41a semana, ocasião em que no serviço em questão, se discute com a paciente a possibilidade de indução ou resolução do parto. Para esta finalidade, analisou-se a distribuição das mulheres conforme a presença ou não de melhora do índice de Bishop, de acordo com a data do parto. Os resultados mostram tendência de melhora no G.C., nos partos realizados antes de 41 semanas, ao passo que esta tendência no grupo E.M. ocorre 
nos partos ultimados na $41^{\text {a }}$ semana. Os resultados, no entanto, não são significativos (Tabela 6).

Analisando estes resultados, não foi possivel atestar o valor do estímulo mamário sobre a maturação da cervix. Como explicações para esta conclusão, pode-se pensar que as participantes, em casa, não tivessem realizado ou fizeram inadequadamente, o estímulo mamário. Ainda que isto seja possível é pouco provável, já que as gestantes foram acompanhadas pelo mesmo obstetra e estavam motivadas, a exemplo de muitas gestantes, a terem seus gestações concluídas, após a 40a semana. Outra hipótese é que o estímulo mamário não determine contração ou o faça inadequadamente, para maturação do colo. Esta eventualidade é aventada por outros autores, que mostraram que algumas mulheres não apresentavam contrações uterinas satisfatórias, mesmo tendo níveis plasmáticos de ocitócitos aumentados durante o estímulo mamário ${ }^{1}$. Estas pacientes podem apresentar menor sensibilidade ou menor número de receptores uterinos para a ocitocina $^{5}$. Mais ainda, admite-se que a contração causada pelo estímulo mamário não seja equivalente àquela causada pelo ocitocina endovenosa ${ }^{6}$.

Diante da escassez de trabalhos internacionais e nacionais não foi possivel confrontar nossos resultados e as dúvidas suscitadas com os dados de outras pesquisas. Novos estudos com o estímulo mamário utilizando grupos maiores de pacientes e sob monitorização tocográfica contínua, poderão esclarecer a real validade da técnica na maturação da cervix.

\section{SUMMARY}

Purpose: to evaluate if the nipple stimulation performed by primigravidae, at 40 weeks, modifies Bishop index.

Method: 64 primigravidae, without clinical or obstetrical complications were studied, in two groups. One group, called nipple stimulation group (N.S.G.) had 29 pregnant women. The other, named control group (C.G.) included 35 pregnant women. The N.S.G. performed the nipple stimulation test, bilaterally, from left to right, for two minutes followed by five minutes of rest, during thirty minutes. The test was done three times a day up to 41 weeks of pregnancy or beginning of labor. Statistical analysis of the results was performed using Student's t test, with $5 \%$ significance.

Results: once nipple stimulation was completed in the N.S.G., it was compared with the C.G. considering time of delivery. The results showed no significant differences between the groups regarding cervix modification, according to the Bishop index.
Conclusions: there were no differences of the Bishop index in primigravidae, with more than 40 weeks of pregnancy, who performed nipple stimulation test, when compared with pregnant women of the control group.

KEY WORDS: Nipple stimulation. Labor induction. Pregnancy: normal.

\section{Referências}

1. Amico JA, Seitchik J, Robinson AG. Studies of oxytocin in plasma of women during hypocontractile labor. J Clin Endocrinol Metab 1984; 58:274-8.

2. Bishop EM. Pelvis scoring for elective induction. Obstet Gynecol 1964; 24:266-8.

3. Eden RD, Sokol RJ, Sorokin Y, Cook HJ, Sheeran G, Chick L. The mammary stimulation test- A predictor of preterm delivery ? Am J Obstet Gynecol 1991; 164:1409-19.

4. Elliott JP, Flaherty DO. The use of breast stimulation to ripen the cervix in term pregnancies. Am J Obstet Gynecol 1983; 145:553-6.

5. Finley BE, Amico J, Castillo RN, Seitchik J. Oxytocin and prolactin responses associated with nipple stimulation contraction stress tests. Obstet Gynecol 1986; 67:836-9.

6. Huddleston JF, Sutliff G, Robinson D. Contraction stress test by intermittent nipple stimulation. Obstet Gynecol 1984; 63:669-73.

7. Leake RD, Fisher DA, Ross M. Oxytocin secretory response to breast stimulation in pregnant women. Am J Obstet Gynecol 1984; 148:259-62.

8. Lenke RR, Nemes JM. Use of nipple stimulation to obtain contraction stress test. Obstet Gynecol $1984 ; 63: 345-8$ 